DOI: $10.17516 / 1997-1397-2021-14-2-204-212$

УДК 517.977.55:536.25

\title{
Asymptotic Behavior of Small Perturbations for Unsteady Motion an Ideal Fluid Jet
}

\author{
Viktor K. Andreev* \\ Institute of Computational Modelling SB RAS \\ Krasnoyarsk, Russian Federation \\ Siberian Federal University \\ Krasnoyarsk, Russian Federation
}

Received 14.11.2020, received in revised form 05.01.2021, accepted 15.02.2021

\begin{abstract}
The stability problem of unsteady rotating circular jet motion of an ideal fluid is reduced to solving an initial-boundary value problem for Poincare-Sobolev type equation with an evolutionary condition on the jet free initial boundary. The solution of this problem is constructed by the method of variables separation. The asymptotic amplitudes behavior perturbations of the free jet boundary at $t \rightarrow \infty$ is found. The results obtained are compared with the known results on the stability of the potential jet motion.
\end{abstract}

Keywords: unsteady motion, free boundary, small perturbations, equations of the Poincare-Sobolev type, instability.

Citation: V.K. Andreev, Asymptotic Behavior of Small Perturbations for Unsteady Motion an Ideal Fluid Jet, J. Sib. Fed. Univ. Math. Phys., 2021, 14(2), 204-212.

DOI: 10.17516/1997-1397-2021-14-2-204-212.

\section{Introduction}

There are numeros works devoted to the study small disturbances of rest or uniform fluid rotation (see $[1,2]$ and the literature available there). Let $\boldsymbol{e}_{3}$ is unit vector of the rotation axis $z$. Homogeneous gravity field of intensity $-g \boldsymbol{e}_{3}$ acts on an ideal fluid. Fluid rotates with constant angular velocity $\omega_{0} \boldsymbol{e}_{3}\left(\omega_{0}>0\right)$. The equations of small oscillations of such a fluid were derived by A. Poincare in 1885 [3]. In a rotating Euler coordinate system, they have the form

$$
\boldsymbol{U}_{t}-\omega_{0} \boldsymbol{e}_{3} \times \boldsymbol{U}+\frac{1}{\rho_{0}} \nabla P=0, \quad \operatorname{div} \boldsymbol{U}=0,
$$

where $\boldsymbol{U}, P$ are speed and pressure disturbances, $\rho_{0}>0$ is constant fluid density. In this case, the main state is $\left(0,0,0,-g z+\omega_{0}^{2}\left(x^{2}+y^{2}\right) / 2\right)$. Poincare showed that any component of the vector $\boldsymbol{U}$ and the function $P$ are the solution of the equation

$$
\frac{\partial^{2}}{\partial t^{2}} \triangle \Psi+4 \omega_{0}^{2} \frac{\partial^{2} \Psi}{\partial z^{2}}=0
$$

where $\triangle$ is Laplace operator in variables $x, y, z$. A survey of the results of A. Poincare and some other authors can be found in the classical monograph [4] (see chapter XII "Rotating masses of liquid").

*andr@icm.krasn.ru

(C) Siberian Federal University. All rights reserved 
The solvability of some initial-boundary value problems for system (0.1) was established by S. L. Sobolev in [5]. He also introduced the state function $\Phi(x, y, z, t)$ in terms of which the solution of system (0.1) is expressed in the form [6]:

$$
\begin{gathered}
\boldsymbol{U}=\frac{\partial^{2}}{\partial t^{2}} \nabla \Phi+2 \omega_{0} \frac{\partial}{\partial t} \nabla \Phi \times \boldsymbol{e}_{3}+4 \omega_{0}^{2}\left(\nabla \Phi \cdot \boldsymbol{e}_{3}\right) \boldsymbol{e}_{3}, \\
\frac{1}{\rho_{0}} P=-\frac{\partial^{3} \Phi}{\partial t^{3}}-4 \omega_{0}^{2} \frac{\partial^{2} \Phi}{\partial t^{2}},
\end{gathered}
$$

and the function $\Phi$ satisfies (0.2). Representation (0.3) makes it possible to more simply establish the properties of solutions of capillary hydrodynamics problems [2]. Equation (0.2) does not satisfy the Cauchy-Kovalevskaya conditions. Its nonclassicality significantly affects the formulation of boundary value problems, the asymptotic properties of solutions, and the properties of the spectrum $[7,8]$. In connection with the above, equation $(0.2)$ is usually called the PoincareSobolev equation. More general differential equations that are not resolved with respect to the highest time derivative are studied in [8]. These include the equations of small perturbations in the atmosphere and ocean [9-11] in particular. In contrast to $(0.2)$, here the coefficients of differential operators can depend on spatial variables.

In the present work, we study the asymptotic behavior of small perturbations of unsteady tension and rotation of a circular jet of an ideal fluid. In this case, the equation coefficients of the Poincare-Sobolev type already depend on the time variable. Using the Lagrange coordinates, it is possible to separate the variables and obtain the amplitude equations. They are ODEs of the second order in the time variable. The asymptotic behavior of the perturbation of the jet free boundary is found. A comparison with a similar behavior of perturbations for a non-rotating jet is made.

\section{Basic motion}

System of equations for an ideal incompressible fluid in Lagrangian coordinates $\boldsymbol{\xi}=(\xi, \eta, \zeta)$ has the form $[12,13]$

$$
\begin{gathered}
M^{*}\left(\mathbf{x}_{t}-\mathbf{g}(\mathbf{x}, t)\right)+\frac{1}{\rho_{0}} \nabla p=0, \\
\operatorname{div} M^{-1} \mathbf{x}_{t}=0,
\end{gathered}
$$

where $M$ is Jacobi matrix of the mapping $\mathbf{x}(\boldsymbol{\xi}, t)$ the initial area $\Omega$ to the region of motion $\Omega_{t}$ at the time $t ; M^{*}, M^{-1}$ are transposed and inverse to $M$ matrices; $\mathbf{g}(\mathbf{x}, t)$ is known vector mass forces; $p(\boldsymbol{\xi}, t)$ is fluid pressure; $\rho_{0}$ is constant density; all differential operations are performed by variables $\xi, \eta, \zeta$. Mass conservation equation (1.2) is equivalent to the equality $\operatorname{det} M=1$ for all $t \geqslant 0[12]$.

Solution of systems (1.1), (1.2) is sought in a fixed region $\Omega$ with initial data a $t=0$

$$
\mathbf{x}=\boldsymbol{\xi}, \quad \mathbf{x}_{t}=\mathbf{u}_{0}(\boldsymbol{\xi}), \quad \operatorname{div} \mathbf{u}_{0}(\boldsymbol{\xi})=0, \quad \boldsymbol{\xi} \in \Omega .
$$

It is easy to check that formulas

$$
\mathbf{x}=M \boldsymbol{\xi}, \quad \frac{1}{\rho_{0}} p=\frac{1}{2}\left(\omega_{0}^{2} \tau-\frac{3}{4} \frac{k^{2}}{\tau^{3}}\right)\left(\xi^{2}+\eta^{2}\right)+f(t)
$$


where

$$
\begin{gathered}
M=\left(\begin{array}{ccc}
\frac{1}{\sqrt{\tau}} \cos \theta & -\frac{1}{\sqrt{\tau}} \sin \theta & 0 \\
\frac{1}{\sqrt{\tau}} \sin \theta & \frac{1}{\sqrt{\tau}} \cos \theta & 0 \\
0 & 0 & \tau
\end{array}\right), \quad \tau=1+k t, \\
k=\text { const }>0, \quad \omega_{0}=\text { const }>0, \quad \theta=\omega_{0} \int_{0}^{\mathrm{t}} \tau(\mathrm{t}) \mathrm{dt},
\end{gathered}
$$

$f(t)$ is an arbitrary function, represent an exact solution of the system (1.1), (1.2) in the absence of mass forces, that is $\mathbf{g}(\mathbf{x}, t)=0$.

If in (1.4) we choose the function $f(t)$ in the form

$$
f(t)=-\frac{1}{2}\left(\omega_{0}^{2} \tau-\frac{3}{4} \frac{k^{2}}{\tau^{3}}\right) r_{0}^{2}+\frac{\sigma \sqrt{\tau}}{r_{0}},
$$

$r_{0}>0, \sigma \geqslant 0$ are constants, then we can give the solution (1.4)-(1.6) next physical interpretation.

At the initial moment of time $t=0$ circular jet of ideal fluid of length $h_{0}$ rotates around the $z$ axis, and vorticity $\boldsymbol{\omega}_{0}=\left(\operatorname{rot} \mathbf{u}_{0}\right) / 2=\left(0,0, \omega_{0}\right)$, see Fig. $1, \mathbf{u}_{0}=\left(-k \xi / 2-\omega_{0} \eta, \omega_{0} \xi-k \eta / 2, k \zeta\right)$.

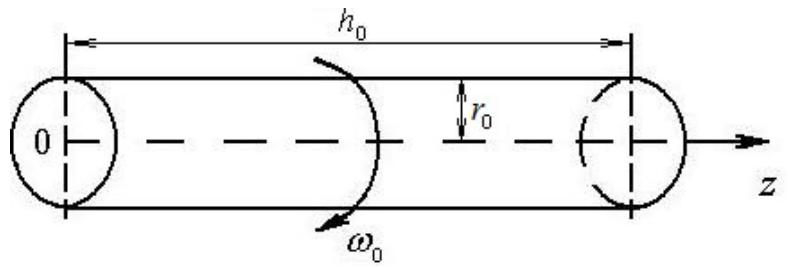

Fig. 1. Motion field scheme

The jet surface $\xi^{2}+\eta^{2}=r_{0}^{2}$ is free and on it capillary forces act ( $\sigma$ is surface coefficient tension). At $t>0$ the jet begins to stretch along the $z$ axis and continues to rotate around this axis. Vorticity in Euler coordinates is $\boldsymbol{\omega}=\operatorname{rot}_{x} \mathbf{u}=\left(0,0, \omega_{0} \tau\right)$ and the velocity vector is

$$
\mathbf{u}=\left(-\frac{k}{2 \tau} x-\omega_{0} \tau y, \omega_{0} \tau x-\frac{k}{2 \tau} y, \frac{k}{\tau} z\right) .
$$

The walls $\zeta=0$ and $\zeta=h_{0}$ can be considered impenetrable, and the second of them at $t=0$ starts to move with the speed $\omega_{0}=$ const. The first wall remains stationary. The moving wall law is $z=(1+k t) h_{0}$, then the constant $k=\omega_{0} / h_{0}$. Free border in all times remain cylindrical, and its radius decreases according to the formula $R(t)=r_{0} / \sqrt{\tau}$.

Remark 1. A solution of the form (1.4) for $\omega_{0}=0$ and $\sigma=0$ was indicated in paper [12] as an example of unsteady motion of an ideal fluid with a free boundary with a linear velocity field. In this case, the matrix $M$ is diagonal, and the movement will be potential.

In Euler coordinates, the pressure is in the form (equality (1.6) taken into account)

$$
\frac{1}{\rho_{0}} p=-\frac{1}{2}\left(\omega_{0}^{2} \tau^{2}-\frac{3}{4} \frac{k^{2}}{\tau^{2}}\right)\left[R^{2}(t)-\left(x^{2}+y^{2}\right)\right]+\frac{\sigma}{R(t)} .
$$

It is clear that the simplest solution (1.7), (1.8) is written in the cylindrical coordinate system $(r, \varphi, z)$, such as,

$$
\mathbf{u}=\left(-\frac{k}{2 \tau} r, \omega_{0} \tau r, \frac{k}{\tau} z\right), \frac{1}{\rho_{0}} p=-\frac{1}{2}\left(\omega_{0}^{2} \tau^{2}-\frac{3}{4} \frac{k^{2}}{\tau^{2}}\right)\left[R^{2}(t)-r^{2}\right]+\frac{\sigma}{R(t)} .
$$




\section{Small perturbation equations}

Since the jet motion described by the formulas (1.4)-(1.6) is vortex, then we will use equations for perturbations in Lagrangian coordinates, obtained in [13]

$$
\begin{gathered}
\operatorname{div}\left[M^{-1} W \int_{0}^{t} W^{-1} M^{*-1}\left(\nabla \Phi+\mathbf{U}_{0}\right) d t_{1}\right]=0, \quad \boldsymbol{\xi} \in \Omega, \quad t>0, \\
\Phi_{t}+a N-\sigma \bar{\Delta}_{\Gamma}(t) N=0, \\
N=b \mathbf{n} \cdot M^{-1} W \int_{0}^{t} W^{-1} M^{*-1}\left(\nabla \Phi+\mathbf{U}_{0}\right) d t_{1}, \quad \boldsymbol{\xi} \in \Gamma, \\
\Phi=0, \quad t=0, \quad \boldsymbol{\xi} \in \Omega \cup \Gamma .
\end{gathered}
$$

We introduced the following notation in expressions (2.1)-(2.3): $M$ is Jacobi matrix of basic motion; $W(\boldsymbol{\xi}, t)$ is solution of matrix equation

$$
W_{t}=\left(\frac{\partial(\mathbf{u})}{\partial(\mathbf{x})}\right)^{*} W,\left.\quad W\right|_{t=0}=\operatorname{diag}(1,1,1),
$$

$\mathbf{U}_{0}(\boldsymbol{\xi})$ is the initial disturbance velocity vector; $\Phi(\boldsymbol{\xi}, t)$ is the disturbance of the Weber's potential $[13]$ and the pressure disturbance is

$$
P(\boldsymbol{\xi}, t)=-\Phi_{t}(\boldsymbol{\xi}, t) .
$$

The quantity $a(\boldsymbol{\xi}, t)$ is given by the equality

$$
a=-\frac{1}{\rho_{0}} \frac{\partial p}{\partial n_{\Gamma_{t}}}-\sigma\left(\frac{1}{R_{1}^{2}}+\frac{1}{R_{2}^{2}}\right)
$$

where $\mathbf{n}_{\Gamma_{t}}$ is the extarnal normal to the boundary of the region of motion $\Omega_{t}, R_{1}, R_{2}$ are the principal radii curvatures of normal sections $\Gamma_{t} ; \bar{\Delta}_{\Gamma}(t)$ is the surface transformation result to the Lagrangian coordinates of the Laplace-Beltrami operator $\Gamma_{t}[13$, c.25],

$$
\begin{gathered}
\bar{\Delta}_{\Gamma}(t)=\frac{1}{q}\left\{\frac{\partial}{\partial \alpha_{1}}\left[\left(G \frac{\partial}{\partial \alpha_{1}}-F \frac{\partial}{\partial \alpha_{2}}\right) q^{-1}\right]+\frac{\partial}{\partial \alpha_{2}}\left[\left(E \frac{\partial}{\partial \alpha_{2}}-F \frac{\partial}{\partial \alpha_{1}}\right) q^{-1}\right]\right\}, \\
E=\left|M \boldsymbol{\xi}_{\alpha_{1}}\right|^{2}, \quad G=\left|M \boldsymbol{\xi}_{\alpha_{2}}\right|^{2}, \quad F=M \boldsymbol{\xi}_{\alpha_{1}} \cdot M \boldsymbol{\xi}_{\alpha_{2}}, \quad q=\left(E G-F^{2}\right)^{1 / 2},
\end{gathered}
$$

$\left(\alpha_{1}, \alpha_{2}\right) \rightarrow \boldsymbol{\xi}\left(\alpha_{1}, \alpha_{2}\right)$ is the parameterization of the initial free boundary $\Gamma$; the function $b(\boldsymbol{\xi}, t)$, $\boldsymbol{\xi} \in \Gamma$ is defined by the equality

$$
b(\boldsymbol{\xi}, t)=\frac{\left|\nabla f_{0}\right|}{\left|M^{*-1} \nabla f_{0}\right|},
$$

$f_{0}(\boldsymbol{\xi})=0$ is the implicit equation on $\Gamma ; \mathbf{n}$ is the external normal to $\Gamma: \mathbf{n}=\nabla f_{0} /\left|\nabla f_{0}\right|$.

The function $N(\boldsymbol{\xi}, t)$ characterizes the deviation of the perturbed boundary from its unperturbed state $\Gamma$ along the normal. Often in stability problems of the fluid motion with a free boundary, this function is the main required quantity $[12,13]$.

The perturbation of the velocity vector by the known $\Phi$ is given by the formula

$$
\mathbf{U}=M\left[M^{-1} W \int_{0}^{t} W^{-1} M^{*-1}\left(\nabla \Phi+\mathbf{U}_{0}\right) d t_{1}\right]_{t}
$$


and vector of perturbations (vector of displacement of liquid particles) is

$$
\mathbf{X}=W \int_{0}^{t} W^{-1} M^{*-1}\left(\nabla \Phi+\mathbf{U}_{0}\right) d t_{1} .
$$

We calculate all auxiliary quantities included in the problem (2.1)-(2.4). In fact it is problem to define a function $\Phi(\boldsymbol{\xi}, t)$.

Using the formulas (1.5) and (1.7), we make sure that the solution of the Cauchy problem (2.5) is the matrix $W \equiv M^{*}$. Further, since the equation $\Gamma$ is $\xi^{2}+\eta^{2}-r_{0}^{2}=0$, and $\Gamma_{t}$ is the equation $x^{2}+y^{2}-r_{0}^{2} / \tau=0$, then $\mathbf{n}=(\xi, \eta, 0) / r_{0}, \mathbf{n}_{\Gamma_{t}}=\tau(x, y, 0) / r_{0}, R_{1}=r_{0} / \sqrt{\tau}, R_{2}=\infty$ and function $a(\boldsymbol{\xi}, t), \boldsymbol{\xi} \in \Gamma$, from formula $(2.7)$ is

$$
a=\frac{r_{0}^{2}}{\rho_{0}}\left(\frac{3}{4} \frac{k^{2}}{\tau^{2}}-\omega_{0}^{2} \tau\right)-\frac{\sigma \tau}{r_{0}^{2}} .
$$

To transform $\bar{\Delta}_{\Gamma}(t)$ from the expression (2.8) we introduce cylindrical Lagrangian coordinates $\rho, \varphi, \zeta$, so that $\xi=\rho \cos \varphi, \eta=\rho \sin \varphi, \zeta=\zeta$. Then for the jet surface $\Gamma$ at the initial moment of time $\alpha_{1}=\varphi, \alpha_{2}=\zeta, 0 \leqslant \varphi \leqslant 2 \pi, 0 \leqslant \zeta \leqslant h_{0}$, a $\rho=r_{0}$. Moreover, the coefficients of the first quadratic form is $E=r_{0}^{2} / \tau, G=\tau^{2}, F=0$ and $q=r_{0} \sqrt{\tau}$, so

$$
\bar{\Delta}_{\Gamma}(t)=\frac{1}{r_{0}^{2} \tau}\left(\tau^{2} \frac{\partial^{2}}{\partial \varphi^{2}}+\frac{r_{0}^{2}}{\tau} \frac{\partial^{2}}{\partial \zeta^{2}}\right) .
$$

Because $f_{0}(\xi, \eta, \zeta)=\xi^{2}+\eta^{2}-r_{0}^{2}=0$, then from the formulas (2.9) and (1.5) we get $b=1 / \sqrt{\tau}$.

Since the matrix $W \equiv M^{*}$, then the equation (2.1) is simplified to the following

$$
\begin{aligned}
& \int_{0}^{t}\left[\tau\left(t_{1}\right) \cos 2\left(\theta(t)-\theta\left(t_{1}\right)\right)\left(\Phi_{\xi \xi}+\Phi_{\eta \eta}\right)+\frac{1}{\tau^{2}\left(t_{1}\right)} \Phi_{\zeta \zeta}\right] d t_{1}= \\
= & \int_{0}^{t}\left[\left(\frac{1}{\tau^{2}\left(t_{1}\right)}-\tau\left(t_{1}\right) \cos 2\left(\theta(t)-\theta\left(t_{1}\right)\right)\right) U_{03 \zeta}+\tau\left(t_{1}\right) \sin 2\left(\theta(t)-\theta\left(t_{1}\right)\right) \Omega_{03}\right] d t_{1},
\end{aligned}
$$

where equality $U_{01 \xi}+U_{02 \eta}+U_{03 \zeta}=0$ was used for initial velocity vector $\mathbf{U}_{0}=\left(U_{01}, U_{02}, U_{03}\right)$; the value $\Omega_{03}=U_{02 \xi}-U_{01 \eta}$ is twice the third component of the initial vortex vector.

Differentiating the equation (2.14) with respect to $t$ three times and introducing the variable $\gamma=\tau^{2}(t)=(1+k t)^{2}$ instead of $t$ we arrive at the equation $\left(\mu=\omega_{0} / k\right)$

$$
\frac{\partial^{2}}{\partial \gamma^{2}}\left(\Phi_{\xi \xi}+\Phi_{\eta \eta}+\frac{1}{\gamma^{3 / 2}} \Phi_{\zeta \zeta}\right)+\frac{\mu^{2}}{\gamma^{3 / 2}} \Phi_{\zeta \zeta}=-\left(\frac{15}{4 \gamma^{7 / 2}}+\frac{\mu^{2}}{\gamma^{3 / 2}}\right) U_{03 \zeta}
$$

The resulting equation must be solved in the initial cylinder $\xi^{2}+\eta^{2}<r_{0}^{2}, 0 \leqslant \varphi \leqslant 2 \pi, 0<\zeta<h_{0}$. In cylindrical coordinates $\rho, \varphi, \zeta$ the equation will be written like this

$$
\frac{\partial^{2}}{\partial \gamma^{2}}\left(\Phi_{\rho \rho}+\frac{1}{\rho} \Phi_{\rho}+\frac{1}{\rho^{2}} \Phi_{\varphi \varphi}+\frac{1}{\gamma^{3 / 2}} \Phi_{\zeta \zeta}\right)+\frac{\mu^{2}}{\gamma^{3 / 2}} \Phi_{\zeta \zeta}=-\left(\frac{15}{4 \gamma^{7 / 2}}+\frac{\mu^{2}}{\gamma^{3 / 2}}\right) U_{03 \zeta} .
$$

Equation (2.15) is an equation of Poincare-Sobolev type (0.2) with variable coefficients and a nonzero right-hand side.

The value $N(\boldsymbol{\xi}, t)$ from the expression (2.3) is converted to the form

$$
\begin{aligned}
N=\frac{1}{r_{0} \sqrt{\tau}} \int_{0}^{t} \tau\left(t_{1}\right)[\cos 2(\theta(t) & \left.-\theta\left(t_{1}\right)\right)\left(\xi \Phi_{\xi}+\eta \Phi_{\eta}+\xi U_{01}+\eta U_{02}\right)+ \\
& \left.+\sin 2\left(\theta(t)-\theta\left(t_{1}\right)\right)\left(\xi \Phi_{\eta}-\eta \Phi_{\xi}+\xi U_{02}-\eta U_{01}\right)\right] d t_{1} .
\end{aligned}
$$


Based on the obtained representation, we write down the equation for the $N$. Differentiating the expression (2.16) twice in $t$ and after some transformations we arrive at the equation

$$
\frac{\partial^{2} \bar{N}}{\partial \gamma^{2}}+\mu^{2} \bar{N}=\frac{1}{2 k} \Phi_{\rho \gamma}+\frac{\mu}{2 k} \Phi_{\varphi}+\frac{\mu}{2 k} V_{0}
$$

where the right-hand side is calculated at $\rho=r_{0}, \bar{N}=\sqrt{\tau} N=\gamma^{1 / 4} N ; V_{0}\left(r_{0}, \varphi, \zeta\right)$ is the azimuthal component of the initial velocity vector in cylindrical coordinates $\left(V_{0}=\cos \varphi U_{02}-\right.$ $\left.\sin \varphi U_{01}\right)$.

As for the boundary condition (2.2) it can be written like this

$$
\Phi_{\gamma}+\frac{1}{2 \gamma^{1 / 4}}\left[\frac{r_{0}^{2} k}{\rho_{0}}\left(\frac{3}{4 \gamma^{3 / 2}}-\mu^{2}\right)-\frac{\sigma}{r_{0}^{2} k}\right] \bar{N}-\frac{\sigma}{2 r_{0} k \gamma^{1 / 4}}\left(\bar{N}_{\varphi \varphi}+\frac{r_{0}^{2}}{\gamma^{3 / 2}} \bar{N}_{\zeta \zeta}\right)=0
$$

at $\rho=r_{0}, 0<\varphi<2 \pi, 0<\zeta<h_{0}$.

The initial data at $\gamma=1$ are

$$
\begin{gathered}
\Phi=0, \quad \xi \in \Omega \cup \Gamma \\
\frac{\partial}{\partial \gamma}\left(\Phi_{\rho \rho}+\frac{1}{\rho} \Phi_{\rho}+\frac{1}{\rho^{2}} \Phi_{\varphi \varphi}+\Phi_{\zeta \zeta}\right)=\frac{3}{2} U_{03 \zeta}-\frac{\mu}{2} \Omega_{03}, \quad \xi \in \Omega \\
\bar{N}=0, \quad \xi \in \Gamma, \quad \bar{N}_{\gamma}=U_{0}\left(r_{0}, \varphi, \zeta\right)
\end{gathered}
$$

where $U_{0}(\rho, \varphi, \zeta)=\cos \varphi U_{01}+\sin \varphi U_{02}$ is radial component of the velocity vector at the initial moment of time.

The conditions for the impermeability of solid walls $\zeta=0$ and $\zeta=h_{0}$ are reduced to the fact that the component $Z$ of the perturbation vector $\mathbf{X}=(X, Y, Z)(2.11)$ is equal to zero. Using the equality (2.11), we get

$$
\Phi_{\zeta}+U_{03}=0, \quad \zeta=0 ; h_{0}, \quad t \geqslant 0
$$

\section{Solution of Small Perturbation Equations}

We are looking for a solution to the problem $(2.15)-(2.20)$ in the form of a Fourier series

$$
\Phi(\rho, \varphi, \zeta, \gamma)=\sum_{n=1}^{\infty} \sum_{m=1}^{\infty} A_{n m}(\gamma) I_{m}\left(\frac{\rho}{r_{0}}\right) \exp (i m \varphi) \cos \left(\frac{n \pi \zeta}{h_{0}}\right)+B(\gamma)
$$

From formula (2.19), we get

$$
A_{n m}(1)=0, \quad B(1)=0 .
$$

In expression (3.1) $I_{m}(x)$ is the Bessel function of the 2nd kind.

Substitution of the solution form (3.1) into the formula (2.15) leads to the equations for the amplitudes $A_{n m}(\gamma)$ :

$$
\left[\left(1-\frac{æ^{2}}{\gamma^{3 / 2}}\right) A_{n m}\right]_{\gamma \gamma}-\frac{\mu^{2} æ^{2}}{\gamma^{3 / 2}} A_{n m}=f(\gamma) \bar{U}_{0 n m}
$$

where

$$
\mathfrak{x}^{2}=\frac{n^{2} \pi^{2} r_{0}^{2}}{h_{0}^{2}}, \quad f(\gamma)=-r_{0}^{2}\left(\frac{15}{4 \gamma^{7 / 2}}+\frac{\mu^{2}}{\gamma^{3 / 2}}\right), \quad \bar{U}_{0 n m}=-\frac{n \pi}{h_{0}} U_{0 n m}
$$


and $U_{0 n m}$ are the coefficients of the Fourier series of the function $U_{03}(\rho, \varphi, \zeta)$ :

$$
U_{03}(\rho, \varphi, \zeta)=\sum_{n=0}^{\infty} \sum_{m=0}^{\infty} U_{0 n m} I_{m}\left(\frac{\rho}{r_{0}}\right) \exp (i m \varphi) \sin \left(\frac{n \pi \zeta}{h_{0}}\right) .
$$

Remark 2. The expressions (3.1) and (3.5) satisfy conditions for the flow around the walls (2.20) at $\zeta=0, \zeta=h_{0}$.

If $\bar{N}_{n m}(\gamma)$ are the coefficients of the Fourier series of the form (3.1) of the function $\bar{N}(\varphi, \zeta, \gamma)$, then from (3.1) and (2.18) we get

$$
\begin{aligned}
B_{\gamma}+a(\gamma) D(\gamma)+\sum_{n=1}^{\infty} \sum_{m=1}^{\infty}\left\{\left[a(\gamma)+b_{n m}(\gamma)\right]\right. & \bar{N}_{n m}(\gamma)+ \\
& \left.+A_{n m \gamma}(\gamma) I_{m}(1)\right\} \exp (i m \varphi) \cos \left(\frac{n \pi \zeta}{h_{0}}\right)=0,
\end{aligned}
$$

where $D(\gamma)$ is the free term in expansion $\bar{N}(\varphi, \zeta, \gamma)$,

$$
\begin{gathered}
a(\gamma)=\frac{1}{2 \gamma^{1 / 4}}\left[\frac{r_{0}^{2} k}{\rho_{0}}\left(\frac{3}{4 \gamma^{3 / 2}}-\mu^{2}\right)-\frac{\sigma}{r_{0}^{2} k}\right], \\
b_{n m}=\frac{\sigma}{2 r_{0} k \gamma^{1 / 4}}\left(m^{2}+\frac{\Re^{2}}{\gamma^{3 / 2}}\right) .
\end{gathered}
$$

Hence,

$$
B_{\gamma}=-a(\gamma) D(\gamma), \quad \bar{N}_{n m}(\gamma)=-\frac{I_{m}(1)}{a(\gamma)+b_{n m}(\gamma)} \frac{d A_{n m}(\gamma)}{d \gamma}
$$

The function $D(\gamma)$ is found from formula $(2.17)$ as a solution of the equation

$$
\frac{d^{2} D}{d \gamma^{2}}+\mu^{2} D=\frac{\mu}{2 k} V_{00}
$$

Let's turn to the equation (3.3), in which we will make the replacement

$$
H=\left(1-\frac{æ^{2}}{\gamma^{3 / 2}}\right) A_{n m}
$$

with $n$ and $m$ are fixed. Then the function $H$ is a solution of the equation

$$
\frac{d^{2} H}{d \gamma^{2}}-\frac{\mu^{2} æ^{2}}{\gamma^{3 / 2}-\aleph^{2}} H=f(\gamma) \bar{U}_{0 n m} .
$$

The fundamental system $H_{1}(\gamma), H_{2}(\gamma)$ of the homogeneous equation (3.11) at $\gamma \gg 1$ has the form [14]

$$
H_{1,2}=\gamma^{3 / 8} \exp \left( \pm 4 \mu æ \gamma^{1 / 4}\right)\left(1+\mathcal{E}_{1,2}(\gamma)\right), \quad \lim _{\gamma \rightarrow \infty} \mathcal{E}_{1,2}(\gamma)=0
$$

These asymptotics can be differentiated twice (see [14, p. 58])

$$
H_{1,2}^{(j)} \sim\left( \pm \frac{1}{\gamma^{3 / 4}}\right)^{(j)} \gamma^{3 / 8} \exp \left( \pm 4 \mu æ \gamma^{1 / 4}\right)
$$

at $\gamma \rightarrow \infty$.

From formula (3.4) function $f(\gamma)$ and relations (3.12), (3.13) we obtain the asymptotic representation of the solution of the equation for large $\gamma(3.11)$ :

$$
H \sim \gamma^{3 / 8}\left[C_{1} \exp \left(4 \mu æ \gamma^{1 / 4}\right)+C_{2} \exp \left(-4 \mu æ \gamma^{1 / 4}\right)\right]+\frac{r_{0}^{2}}{æ^{2}} .
$$


According to the replacement (3.10) we have $A_{n m} \sim H$ at $\gamma \rightarrow \infty$. Then from expressions (3.8), (3.13) and (3.14) we will find

$$
\bar{N}_{n m}(\gamma) \sim \gamma^{-9 / 8} \exp \left(4 \mu æ \gamma^{1 / 4}\right), \quad \gamma \rightarrow \infty .
$$

Moreover, the asymptotics does not depend on the surface tension coefficient at $\mu \neq 0$. Since $\bar{N}_{n m}(\gamma)=\gamma^{1 / 4} N_{n m}$, then for the amplitudes of perturbations of the free boundary we obtain from the formula (3.15)

$$
N_{n m} \sim \gamma^{-11 / 8} \exp \left(4 \mu æ \gamma^{1 / 4}\right), \quad \gamma \rightarrow \infty .
$$

The function $D(\gamma)$ is found from the equation $(3.9)$ :

$$
D=C_{3} \cos \mu \gamma+C_{4} \sin \mu \gamma+\frac{V_{00}}{2 k \mu},
$$

then from formula (3.8)

$$
B(\gamma)=-\int_{1}^{\gamma} a(\tau)\left[C_{3} \cos \mu \tau+C_{4} \sin \mu \tau+\frac{V_{00}}{2 k \mu}\right] d \tau .
$$

Thus, there is an instability.

\section{Conclusion}

The problem on the behavior of small perturbations of an expanding and rotating jet of an ideal fluid is reduced to a problem for equations of the Poincare-Sobolev type with variable coefficients depending on time. Due to the specifics of complex boundary conditions and the equation itself, the solution was found by the method of variables separation. For the obtained amplitude equations, the asymptotic of the solutions is found for $t \rightarrow \infty$. Since $\gamma \sim(k t)^{2}$, then from (3.16) we get $N_{n m} \sim(k t)^{-11 / 4} \exp \left[4 \mu æ(k t)^{1 / 2}\right]$ at $t \rightarrow \infty$. Notice, that $\mu æ=\left(n \omega_{0} \pi r_{0}\right) /\left(k h_{0}\right)$. Therefore, the growth of perturbations of the free boundary does not depend on the influence of capillarity (parameter $\sigma$ ), as well as on the parameter $m$. For "pure" stretching of the jet $\left(\omega_{0}=0\right)$, the results differ significantly. Namely, [13], at $\sigma=0 N_{n 0} \sim(k t)^{-1 / 2}, N_{n m} \sim(k t)^{1 / 2}$, $m \geqslant 1$, and at $\sigma \neq 0 N_{n 0} \sim(k t)^{-5 / 8} \exp \left[2 \pi n h_{0} r_{0}^{-1} \sqrt{\mathrm{We}}(k t)^{1 / 4}\right], N_{n m} \sim(k t)^{-1 / 8}(m \geqslant 1)$, where $\mathrm{We}=\sigma /\left(\rho r_{0}^{3} k^{2}\right)$ is the Weber number. Thus, here the surface tension has very strong effects on the perturbations behavior, especially for axisymmetric perturbations with $m=0$.

This research was supported by the Krasnoyarsk Mathematical Center and financed by the Ministry of Science and Higher Education of the Russian Federation in the framework of the establishment and development of regional Centers for Mathematics Research and Education (Agreement no. 075-02-2020-1631).

\section{References}

[1] N.N.Moiseev, V.V.Rumyantsev, Dinamika tela s polostyami, soderzhachshimi zhidkost' (Dynamics of Bodies with Fluid-Filled Cavities), Moscow, Nauka, 1965 (in Russian).

[2] V.G.Babskii, N.D.Kopachevskii, A.D.Myshkis, L.A.Slobozhanin, A.D.Tyuptsov, LowGravity Fluid Mechanics, Moscow, Nauka, 1976 (in Russian). 
[3] A.Poincaré, Sur l'équilibre d'une masee fluide animée d'un mouvement de rotation, Acta Math., 7(1985), 259-380.

[4] H.Lamb, Hydrodynamics, Moscow, Leningrad, Gostekhizdat, 1947 (in Russian).

[5] S.L.Sobolev, On a new problem of mathematical physics, Izv. Akad. Nauk SSSR Ser. Mat., 18(1954), no. 1, 3-50.

[6] S.L.Sobolev, On Symmetric Top Motion with a Fluid-Filled Cavity, Prikl. Mekh. Tekh. Fiz., 3(1960), 20-55 (in Russian).

[7] T.I. Zelenyak, Selected Problems of the Qualitative Theory of Partial Differential Equations, Novosibirsk Univ., Novosibirsk, 1970 (in Russian).

[8] S.V.Uspenskij, G.V.Demidenko, V.G.Perepelkin, Imbedding theorems and applications to differential equations, Nauka, Novosibirsk, 1984 (in Russian).

[9] V.M.Kamenkovich, A.S.Monin, Ocean Physics, Moscow, Nauka, 1978 (in Russian).

[10] M.J.Lighthill, On waves generated in dispersive systems by travelling forcing effects, with applications to the dynamics of rotating fluids, Jour. Fluid Mech., 27(1967), no. 4, 725-752.

[11] H.P.Greenspan, On the transient motion of a contained rotatinh fluid, Jour. Fluid Mech., 20(1964), no. 4, 673-696.

[12] L.V.Ovsyannikov, General equations and examples, Problems of Unsteady Free-Boundary Fluid Flows, Nauka, Novosibirsk, 1967, 5-75 (in Russian).

[13] V.K.Andreev, Stability of unsteady fluid motions with a free boundary, Novosibirsk, Nauka, 1992 (in Russian).

[14] M.V.Fedoryuk, Asymptotic Methods for Linear Ordinary Differential Equations, Moscow, Nauka, 1983 (in Russian).

\title{
Асимптотическое поведение малых возмущений нестационарного движения струи идеальной жидкости
}

Виктор К. Андреев

Институт вычислительного моделирования СО РАН

Красноярск, Российская Федерация Сибирский федеральный университет

Красноярск, Российская Федерация

\begin{abstract}
Аннотация. Задача об устойчивости нестационарного движения вращающейся круглой струи идеальной жидкости сведена к решению начально-краевой задачи для уравнения типа ПуанкареСоболева с эволюционным условием на свободной начальной границе струи. Решение поставленной задачи построено методом разделения переменных. Найдено асимптотическое поведение амплитуд возмущения свободной границы струи при $t \rightarrow \infty$. Произведено сравнение полученных результатов с известными результатами об устойчивости потенциального движения струи.
\end{abstract}

Ключевые слова: нестационарное движение, свободная граница, малые возмущения, уравнения типа Пуанкаре-Соболева, неустойчивость. 